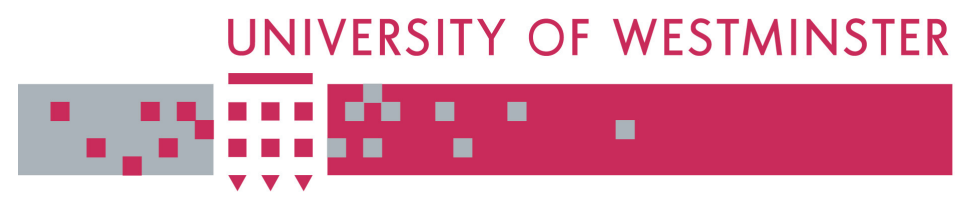

WestminsterResearch

http://www.wmin.ac.uk/westminsterresearch

\title{
Spectral analysis of randomly sampled signals: suppression of aliasing and sampler jitter.
}

\author{
Andrzej Tarczynski \\ Najib Allay
}

Cavendish School of Computer Science

Copyright (C [2004] IEEE. Reprinted from IEEE Transactions on Signal Processing, 52 (12). pp. 3324-3334.

This material is posted here with permission of the IEEE. Such permission of the IEEE does not in any way imply IEEE endorsement of any of the University of Westminster's products or services. Internal or personal use of this material is permitted. However, permission to reprint/republish this material for advertising or promotional purposes or for creating new collective works for resale or redistribution must be obtained from the IEEE by writing to pubs-permissions@ieee.org. By choosing to view this document, you agree to all provisions of the copyright laws protecting it.

The WestminsterResearch online digital archive at the University of Westminster aims to make the research output of the University available to a wider audience. Copyright and Moral Rights remain with the authors and/or copyright owners. Users are permitted to download and/or print one copy for non-commercial private study or research. Further distribution and any use of material from within this archive for profit-making enterprises or for commercial gain is strictly forbidden.

Whilst further distribution of specific materials from within this archive is forbidden, you may freely distribute the URL of WestminsterResearch.

(http://www.wmin.ac.uk/westminsterresearch).

In case of abuse or copyright appearing without permission e-mail wattsn@wmin.ac.uk. 


\title{
Spectral Analysis of Randomly Sampled Signals: Suppression of Aliasing and Sampler Jitter
}

\author{
Andrzej Tarczynski, Member, IEEE, and Najib Allay
}

\begin{abstract}
Nonuniform sampling can facilitate digital alias-free signal processing (DASP), i.e., digital signal processing that is not affected by aliasing. This paper presents two DASP approaches for spectrum estimation of continuous-time signals. The proposed algorithms, named the weighted sample (WS) and weighted probability (WP) density functions, respectively, utilize random sampling to suppress aliasing. Both methods produce unbiased estimators of the signal spectrum. To achieve this effect, the computational procedure for each method has been suitably matched with the probability density function characterising the pseudorandom generators of the sampling instants. Both proposed methods are analyzed, and the qualities of the estimators they produce have been compared with each other. Although none of the proposed spectrum estimators is universally better than the other one, it has been shown that in practical cases, the WP estimator produces generally smaller errors than those obtained from WS estimation. A practical limitation of the approaches caused by the sampling-instant jitter is also studied. It has been proven that in the presence of jitter, the theoretically infinite bandwidths of WS and WP signal analyses are limited. The maximum frequency up to which these analyses can be performed is inversely proportional to the size of the jitter.
\end{abstract}

Index Terms-Alias-free estimation, Fourier transform, nonuniform sampling, sampling methods, spectral analysis.

\section{INTRODUCTION}

D IGITAL alias-free signal processing (DASP) can only be performed when the processed signals are sampled at nonuniformly distributed sampling instants. However, usage of nonuniform sampling is merely a necessary and by no means a sufficient condition for practicing DASP. Therefore, it is not surprising that, despite the fact that the theory of processing nonuniformly sampled signals has been studied for a number of decades [1]-[3], DASP methodology has attracted only a small fraction of the interest devoted to that area.

There are a number of reasons why nonuniform sampling is used in signal processing. In some cases, this is enforced by inconvenient circumstances such as inaccessibility of the measured signals at some time instants or periods of time, as often happens in astronomy, geophysical sciences, or medicine [4]. Another cause of nonuniform sampling is when some samples are lost from otherwise uniformly distributed sampling sequences [5]. Nonuniform sampling can also be a means of com-

Manuscript received November 13, 2002; revised November 13, 2003. This work was supported in part by the IST-2001-34552 project funded by the European Commission. The associate editor coordinating the review of this manuscript and approving it for publication was Prof. Jian Li.

The authors are with the Department of Electronic Systems, University of Westminster, London, W1W 6UW, U.K. (e-mail: tarczya@wmin.ac.uk; allayn@cmsa.wmin.ac.uk).

Digital Object Identifier 10.1109/TSP.2004.837436 pressing data. Compression can be achieved by relating the sampling rate with the signal rate and, hence, reducing the storage space over the periods when the signal changes slowly [6]. In the case of DASP, nonuniform sampling is introduced intentionally to provide additional flexibility and opportunities, which are not available when the traditional periodic sampling schemes are in use. As a general rule, the sampling schemes used here are based on random sampling independent of the shape of the processed signal [7], [8]. One of the earliest ideas of DASP can be traced back to [8], where Shapiro and Silverman proposed sampling schemes and processing algorithms that allowed estimation of the power spectral density of random stationary signals. Since then, several authors have reconsidered and extended DASP techniques [9]-[11], and some interesting applications in instrumentation [12] and digital radio [13] have been found. In general, processing of nonuniformly sampled signals is more complicated and time-consuming than classical DSP. The main benefit of using DASP is that these techniques often enable digital signal analysis to be performed over a wide range of frequencies while maintaining a low sampling rate. Therefore, DASP can be recommended mainly for processing signals that, due to technical or economical constraints, cannot be sampled fast enough to facilitate usage of classical DSP. Interesting reviews of DASP techniques can be found in [7], [13], and [14].

This paper builds on the earlier results reported in [15] and [16]. We propose two DASP algorithms for spectral analysis of signals. The spectrum is understood here as the complex-valued Fourier transform of the signal. The frequency bandwidth within which the proposed method can be used to estimate the signal spectrum exceeds significantly the traditional Nyquist limit of half of the sampling rate.

The problem of estimating spectrum from nonuniformly distributed samples has a relatively long history. Below, we discuss briefly how our work relates to some major approaches to this problem.

Early DASP papers [8], [9] aimed at reconstructing the power spectral density of random stationary signals using infinitely long sequences of nonuniformly distributed signal samples. Here, we tackle deterministic signals and estimate the Fourier transform using only finite numbers of signal samples.

Lomb [17] and Scargle [18], [19] investigated the properties of the periodogram obtained from arbitrarily distributed signal samples and its use in detecting the discrete spectral components of the analyzed signal. Their research was motivated by applications where nonuniform sampling is enforced by the experimental circumstances, and freedom in selecting sampling instants is limited. The methods proposed in our paper rely 
on the user's ability to sample the signal at prescribed sampling instants. The signals are sampled nonuniformly but not arbitrarily. The algorithms that process the acquired data are matched with the properties of the sampling instant generators. Consequently, with the use of the methods proposed here, it is possible to tell more about the accuracy of spectrum estimation than in [17]-[19]. Of course, all the results related to the periodogram remain valid for the sampling schemes that we suggest in this paper. The periodogram obtained from nonuniformly distributed samples may show alias-free properties. However, when arbitrary sampling is used, alias-free features cannot be guaranteed, as their existence depends on the use of suitable sampling schemes. Another difference between the approaches is that the periodogram represents only the magnitude of the signal spectrum, whereas the WS and WP algorithms estimate the complex-valued Fourier transform of the signal, allowing extraction of not only the magnitude of the spectrum but also its phase.

The approach presented in this paper is related to the concept of DASP developed by Bilinskis [7], [11], [20] and followed by others [15], [16], [21], [22]. This paper contributes a few new results to the theory of DASP. First, we show that alias-free DSP can be performed even when the sampling point density function varies with time. In fact, we show that by introducing suitable variations of the sampling point density function, it is possible to obtain a more accurate estimate of the signal spectrum than in the case where the concentration of the samples is uniform across the window. This observation generalizes the recommendation formulated in [7], which stipulates that DASP uses sampling schemes producing a constant point density function. Second, we construct unbiased estimators of the signal spectrum for the case when the sampling instants are subjected to random jitter. Third, we provide tools for assessing accuracy of spectrum approximation in all considered cases by deriving closed-form formulas for standard deviations of the estimators.

This paper is organized as follows. In Section II, we define the target signal spectrum that is to be estimated using signal samples. In Section III, we define the WS and WP estimators of the spectrum. We prove that both are unbiased. In the next section, we derive the standard deviations of the estimators. The qualities of both estimators are compared with each other in Section V. Section VI presents simulation results of spectrum estimation using the proposed approaches. In Section VII, we discuss the effect of sampling instant jitter on the quality and achievable bandwidth of spectrum estimation. Finally, in Section VIII, we provide numerical analysis of sampling instant jitter.

\section{TARget Spectrum of Continuous-Time Signals}

The spectrum of a deterministic signal is defined by its Fourier transform [23]

$$
X(f) \hat{=} \int_{-\infty}^{\infty} x(t) \exp (-j 2 \pi f t) d t
$$

The above definition is rarely used in practical situations since it requires that the analyzed signal is known in an infinitely long time interval. Therefore, in practice, spectrum (1) is often replaced with its truncated version

$$
X_{T}(f) \hat{=} \int_{0}^{T} x(t) \exp (-j 2 \pi f t) d t
$$

where $T$ is the length of the time interval over which signal $x(t)$ is observed. Owing to the Gibbs phenomenon, the shape of spectrum $X_{T}(f)$ may be significantly different from $X(f)$ in the neighborhood of frequencies where $X(f)$ is discontinuous. A standard solution for suppressing the Gibbs phenomenon is to smooth out those differences by using a nonrectangular window $w(t)$

$$
X_{W}(f) \hat{=} \int_{0}^{T} x(t) w(t) \exp (-j 2 \pi f t) d t .
$$

There is a vast choice of windows that can be used in (3). An excellent review of the topic that includes analysis of their properties and smoothing effects can be found in [23]. The research in this area is still going on, and from time to time, new additions are being reported, e.g., [24]. In this paper, we do not insist on using any particular $w(t)$. The decisions about the shape of the window $w(t)$ and its length $T$ are left to the user. The main task that we tackle here is to estimate the spectrum (3) of signal $x(t)$ by acquiring and processing signal samples.

The results presented in this paper are valid for any window $w(t)$, with a minor exception that in the WP case the window $w(t)$ must not take negative values. Additional constraints on $w(t)$ are imposed when we compare the quality of the proposed WS and WP estimators. These comparisons are performed under the following assumptions.

a) $w(t)$ is Lipschitz [25], i.e. $\left|w\left(t_{1}\right)-w\left(t_{2}\right)\right| \leq C\left|t_{1}-t_{2}\right|$, where $C$ is a small constant.

b) $w(t)$ achieves its maximum in the middle of the interval $[0, T]$.

c) $w(t)$ decreases monotonically (but not necessarily strictly monotonically) toward the ends of this interval.

These limitations do not exclude any popularly used windows and, therefore, are not too restrictive.

\section{UNBIASED SPECTRUM ESTIMATORS}

The names of the two methods for spectrum estimation [weighted samples (WS) and weighted probability (WP) density function] reflect the way in which the shape of the window $w(t)$ is taken into account. Since both methods use randomly selected sampling instants, the spectrum estimators they produce are random variables. In the following two subsections, we define both estimators and prove that they are unbiased for any frequency $f$. This observation justifies our claim that the techniques we propose here belong to DASP.

\section{A. WS Method}

The sampling instants used in the WS approach are identical random variables that are independent from each other, whose 
probability density function (PDF) is uniformly distributed inside the observation window.

$$
p_{\mathrm{WS}}(t) \hat{=} \begin{cases}\frac{1}{T}, & \text { when } t \in[0, T] \\ 0, & \text { when } t \notin[0, T] .\end{cases}
$$

The spectrum of the signal is estimated using the following formula:

$$
X_{\mathrm{WS}}(f) \hat{=} \frac{T}{N} \sum_{n=1}^{N} x\left(t_{n}\right) w\left(t_{n}\right) \exp \left(-j 2 \pi f t_{n}\right) .
$$

We prove that this estimator is unbiased for any frequency $f$, i.e., its expected value is $X_{W}(f)$ defined by (3). Note that all components of the sum in (5) are random variables with identical PDFs. The expected value of each component is

$$
X_{\mathrm{WS}}^{C}(f) \hat{=} \mathrm{E}\{x(t) w(t) \exp (-j 2 \pi f t)\}
$$

where $t$ is a random variable whose PDF is described by (4). The right-hand side of (6) can be calculated as follows: $X_{\mathrm{WS}}^{C}(f)=$ $\int_{-\infty}^{\infty} x(t) w(t) \exp (-j 2 \pi f t) p_{\mathrm{WS}}(t) d t$, implying that

$$
X_{\mathrm{WS}}^{C}(f)=\frac{1}{T} \int_{0}^{T} x(t) w(t) \exp (-j 2 \pi f t) d t=\frac{1}{T} X_{W}(f) .
$$

By using (5)-(7), we obtain

$$
E\left\{X_{\mathrm{WS}}(f)\right\}=X_{W}(f)
$$

which completes the proof that $X_{\mathrm{WS}}(f)$ is unbiased.

\section{B. WP Method}

The sampling instants used in the WP approach are also random variables that are independent from each other with identical PDF. However, in this case, the density of sampling instants is proportional to $w(t)$. The PDF is given by

$$
p_{\mathrm{WP}}(t) \hat{=} \begin{cases}\frac{1}{A T} w(t), & \text { when } t \in[0, T] \\ 0, & \text { when } t \notin[0, T] .\end{cases}
$$

Constant $A$ denotes the average value of $w(t)$ inside the interval $[0, T]$

$$
A \hat{=} \frac{1}{T} \int_{0}^{T} w(t) d t
$$

The scaling factor $1 / A T$ guarantees that $p_{\mathrm{WP}}(t)$ satisfies one of the axioms of PDF: $\int_{-\infty}^{\infty} p_{\mathrm{WP}}(t) d t=1$. It follows from (9) that $w(t)$ must not be negative. The WP estimator of the signal spectrum is defined by

$$
X_{\mathrm{WP}}(f) \hat{=} \frac{A T}{N} \sum_{n=1}^{N} x\left(t_{n}\right) \exp \left(-j 2 \pi f t_{n}\right)
$$

To prove that $X_{\mathrm{WP}}(f)$ is unbiased, we calculate its expected value. Similarly to the previous case, the sum in (11) consists of $N$ identically distributed random variables. The expected value of each component is

$$
\begin{aligned}
X_{\mathrm{WP}}^{C}(f) & \hat{=} E\{x(t) \exp (-j 2 \pi f t)\} \\
& =\int_{-\infty}^{\infty} x(t) \exp (-j 2 \pi f t) p_{\mathrm{WP}}(t) d t .
\end{aligned}
$$

Therefore

$$
X_{\mathrm{WP}}^{C}(f)=\frac{1}{A T} \int_{0}^{T} x(t) \exp (-j 2 \pi f t) w(t) d t=\frac{1}{A T} X_{W}(f)
$$

and

$$
E\left\{X_{\mathrm{WP}}(f)\right\}=X_{W}(f) .
$$

This completes the proof that $X_{\mathrm{WP}}(f)$ is also an unbiased estimator of $X_{W}(f)$.

\section{ACCURACY OF SPECTRUM ESTIMATION}

Lack of bias is an important and sought-after property of any statistical estimator. However, unbiased estimators can still be inaccurate. It follows from the Bienayme-Chebyshev inequality [25] that the size of estimation error is closely related to the standard deviation of the estimator. In the subsequent analyzes, we calculate the standard deviation $\sigma_{X}$ of a random variable $X$ as the square root of its variance $\sigma_{X}^{2}=\mathrm{E}\left\{|X|^{2}\right\}-|\mathrm{E}\{X\}|^{2}$.

\section{A. Standard Deviation of the WS Estimator}

The variance of $X_{\mathrm{WS}}(f)$ is given by

$$
\sigma_{X_{\mathrm{WS}}}^{2}(f)=\mathrm{E}\left\{\left|X_{\mathrm{WS}}(f)\right|^{2}\right\}-\left|X_{W}(f)\right|^{2} .
$$

Now, $\left|X_{\mathrm{WS}}(f)\right|^{2}=(T / N)^{2}\left(\sum_{n=1}{ }^{N} x^{2}\left(t_{n}\right) w^{2}\left(t_{n}\right)+\sum_{n=1}^{N} \sum_{\substack{k=1 \\ n \neq k}}^{N}\right.$ $\left.\left\{x\left(t_{n}\right) w\left(t_{n}\right) \exp \left(-j 2 \pi f t_{n}\right)\right\}\left\{x\left(t_{k}\right) w\left(t_{k}\right) \exp \left(j 2 \pi f t_{k}\right)\right\}\right)$.

Since the sampling instants bearing distinct indices are independent from each other, we can apply (6) and (7) to the formula above to get

$$
\begin{aligned}
\mathrm{E}\left\{\left|X_{\mathrm{WS}}(f)\right|^{2}\right\}=\left(\frac{T}{N}\right)^{2} \\
\times\left[N \mathrm{E}\left\{x^{2}(t) w^{2}(t)\right\}+\frac{N(N-1)}{T^{2}}\left|X_{W}(f)\right|^{2}\right] .
\end{aligned}
$$

Note that $E\left\{x^{2}(t) w^{2}(t)\right\}=\int_{-\infty}^{\infty} x^{2}(t) w^{2}(t) p_{\mathrm{WS}}(t) d t=$ $(1 / T) \int_{0}^{T} x^{2}(t) w^{2}(t) d t$. Therefore

$$
\mathrm{E}\left\{x^{2}(t) w^{2}(t)\right\}=\frac{1}{T} E_{\mathrm{WS}}
$$


where $E_{\mathrm{WS}}$ is the energy of the windowed signal

$$
E_{\mathrm{WS}} \hat{=} \int_{0}^{T}[x(t) w(t)]^{2} d t .
$$

By combining (16)-(18), we obtain

$$
\mathrm{E}\left\{\left|X_{\mathrm{WS}}(f)\right|^{2}\right\}=\frac{T}{N} E_{\mathrm{WS}}+\frac{N-1}{N}\left|X_{W}(f)\right|^{2} .
$$

After substituting (19) into (15), we get

$$
\sigma_{X_{\mathrm{WS}}}^{2}(f)=\frac{T E_{\mathrm{WS}}-\left|X_{W}(f)\right|^{2}}{N}
$$

and finally

$$
\sigma_{X_{\mathrm{WS}}}(f)=\sqrt{\frac{T E_{\mathrm{WS}}-\left|X_{W}(f)\right|^{2}}{N}} .
$$

\section{B. Standard Deviation of WP Estimator}

The procedure for deriving the variance and standard deviation of $X_{\mathrm{WP}}(f)$ is similar to what we presented in the previous subsection. Therefore, we omit the details of calculations and present only the final results. The variance of $X_{\mathrm{WP}}(f)$ is given by

$$
\sigma_{X_{\mathrm{WP}}}^{2}(f)=\frac{A T E_{\mathrm{WP}}-\left|X_{W}(f)\right|^{2}}{N}
$$

while its standard deviation is

$$
\sigma_{X_{\mathrm{WP}}}(f)=\sqrt{\frac{A T E_{\mathrm{WP}}-\left|X_{W}(f)\right|^{2}}{N}} .
$$

The weighted energy $E_{\mathrm{WP}}$ in (22) and (23) is defined by

$$
E_{\mathrm{WP}} \hat{=} \int_{0}^{T} x^{2}(t) w(t) d t .
$$

The standard deviations of $X_{\mathrm{WS}}(f)$ and $X_{\mathrm{WP}}(f)$ are inversely proportional to $\sqrt{N}$. This suggests that the estimation errors decrease at this rate when, for a fixed window length $T$, we increase the number of samples. Since $\left|X_{W}(f)\right| \geq 0$, we can use (21) and (23) to formulate the upper bounds for the appropriate standard deviations: $\sigma_{\mathrm{WS}}(f) \leq \sigma_{\mathrm{WS}, \max }=$ $\sqrt{\left(T E_{\mathrm{WS}}\right) / N}$ and $\sigma_{\mathrm{WP}}(f) \leq \sigma_{\mathrm{WP}, \max }=\sqrt{\left(A T E_{\mathrm{WP}}\right) / N}$. The bounds $\sigma_{\mathrm{WS} \text {, max }}$ and $\sigma_{\mathrm{WP} \text {,max }}$ indicate the average spectrum estimation errors at frequencies where the actual spectrum of the signal is zero. These white-noise-like errors can be clearly visible in the estimated spectra, as demonstrated in the numerical examples in Section VI. The bounds $\sigma_{\mathrm{WS} \text {,max }}$ and $\sigma_{\mathrm{WP}, \max }$ also allow us to assess the threshold levels below which the spectral components of the analyzed signals cannot be detected by straightforward use of the proposed approaches.

The presence of unstructured noise in the estimated spectrum can be explained by the fact that nonuniformly sampled sinusoids are usually not orthogonal. Therefore, even when the analyzed signal is a sinusoid, the spectral estimators (5) and (11) give nonzero results for practically any frequency $f$. More information about this topic can be found in [21].

\section{QUALITY COMPARISON FOR SPECTRUM ESTIMATORS}

The analysis of the WS and WP estimators that we performed so far does not give clues about their relative quality. Both estimators are unbiased and the levels of errors they produce are expressed by similar formulas. In this section, we compare the estimators to find out which of them provides more accurate estimates of the spectrum. It follows from (21) and (23) that the simplest way to accomplish this task is to analyze the relation between $E_{\mathrm{WS}}$ and $A E_{\mathrm{WP}}$. If $E_{\mathrm{WS}}<A E_{\mathrm{WP}}$, then $\sigma_{X_{\mathrm{WS}}}(f)<$ $\sigma_{X_{\mathrm{WP}}}(f)$, and consequently, WS is more accurate than WP. In the converse case $E_{\mathrm{WS}}>A E_{\mathrm{WP}}$, we get $\sigma_{X_{\mathrm{WS}}}(f)>\sigma_{X_{\mathrm{WP}}}(f)$, and WP yields smaller errors than WS. In Section V-A below, we present two simple examples showing, in turn, that each of the methods can prevail. Then, in Section V-B, we prove that if the energy of the signal is uniformly distributed inside the interval $[0, T]$, then the WP estimator is more accurate than WS.

\section{A. Comparison of Estimators' Quality for Signals with Nonuniformly Distributed Energy}

In both examples presented in this subsection, we use a triangular window of length $T=1 \mathrm{~s}$ described by

$$
w(t)= \begin{cases}2 t, & \text { when } t \in[0,0.5] \\ 2-2 t, & \text { when } t \in[0.51] .\end{cases}
$$

The average value of $w(t)$ inside the interval $[0,1]$ is $A=0.5$.

Example 1: Consider a signal whose energy is concentrated in the middle of the interval $[0,1]$.

$$
x_{1}(t)= \begin{cases}1, & \text { when } t \in(0.25,0.75) \\ 0, & \text { when } t \in[0,0.25] \cup[0.75,1] .\end{cases}
$$

Using (18) and (24), we get $E_{\mathrm{WS}}=\int_{0.25}^{0.5}(2 t)^{2} d t+\int_{0.5}^{0.75}(2-$ $2 t)^{2} d t=7 / 24$ and $E_{\mathrm{WP}}=\int_{0.25}^{0.5} 2 t d t+\int_{0.5}^{0.75}(2-2 t) d t=3 / 8$. Hence, $A E_{\mathrm{WP}}=3 / 16$, and $E_{\mathrm{WS}}>A E_{\mathrm{WP}}$. Therefore, in this case ,WP spectrum estimation is more accurate than WS.

Example 2: In this example, we select a signal whose energy is concentrated near the borders of the interval $[0,1]$.

$$
x_{2}(t)= \begin{cases}0, & \text { when } t \in(0.25,0.75) \\ 1, & \text { when } t \in[0,0.25] \cup[0.75,1] .\end{cases}
$$

This time, $E_{\mathrm{WS}}=\int_{0}^{0.25}(2 t)^{2} d t+\int_{0.75}^{1}(2-2 t)^{2} d t=1 / 24$, $E_{\mathrm{WP}}=\int_{0}^{0.25} 2 t d t+\int_{0.75}^{1}(2-2 t) d t=1 / 8$, and $A E_{\mathrm{WP}}=$ $1 / 16$. Hence, $E_{\mathrm{WS}}<A E_{\mathrm{WP}}$, and consequently, the WS estimator is more accurate than the WP.

The main conclusion from this simple analysis is that neither of the estimators is universally more accurate than the other one. In general, for windows satisfying assumptions b) and c) from Section II, WP estimation tends to be more accurate for signals with energy concentrated in the central part of the observation window, whereas the WS approach prevails in the less popular cases when most of the signal energy is concentrated at the ends of the window.

\section{B. Comparison of Estimators' Quality for Signals with Uniformly Distributed Energy}

In this section, we present analysis aimed at comparing the accuracy of both estimators for signals whose energy is approx- 
imately uniformly distributed inside the observation window. In order to formalize the idea of uniform distribution of energy, we select a large integer $K$ and divide the interval $[0, T]$ into $K$ short subintervals of length $\Delta=T / K$. We assume that the energy of the signal inside each subinterval is approximately the same, i.e., there exists a constant $E$ such that

$$
\int_{k \Delta}^{(k+1) \Delta} x^{2}(t) d t \approx E
$$

for $k \in\{0,1, \cdots, K-1\}$. According to assumption a), $w(t)$ is a Lipschitz function. Therefore, we can use the following approximation:

$$
\int_{k \Delta}^{(k+1) \Delta} x^{2}(t) w^{2}(t) d t \approx E w^{2}(k \Delta) .
$$

To justify the above statement, we note that for any $t \in$ $[k \Delta,(k+1) \Delta]$, we have $|w(t)-w(k \Delta)| \leq C|t-k \Delta| \leq C \Delta$. Since $w(t)$ is a non-negative function, this inequality implies that $\left|w^{2}(t)-w^{2}(k \Delta)\right|=\mid[w(t)-w(k \Delta)]+$ $2 w(k \Delta)|\times| w(t)-w(k \Delta) \mid \leq C^{2} \Delta^{2}+2 C \Delta w(k \Delta)=$ $O(C \Delta)$. Therefore, $w^{2}(k \Delta)-O(C \Delta) \leq w^{2}(t) \leq$ $w^{2}(k \Delta)+O(C \Delta)$, and $\int_{k \Delta}^{(k+1) \Delta} x^{2}(t)\left[w^{2}(k \Delta)-O(C \Delta)\right] d t \leq$ $\int_{k \Delta}^{(k+1) \Delta} x^{2}(t) w^{2}(t) d t \leq \int_{k \Delta}^{(k+1) \Delta} x^{2}(t)\left[w^{2}(k \Delta)+O(C \Delta)\right] d t$. By substituting (25) in the above formula, we get $E\left[w^{2}(k \Delta)-\right.$ $O(C \Delta)] \leq \int_{k \Delta}^{(k+1) \Delta} x^{2}(t) w^{2}(t) d t \leq E\left[w^{2}(k \Delta)+O(C \Delta)\right]$, and consequently, $\left|E w^{2}(k \Delta)-\int_{k \Delta}^{(k+1) \Delta} x^{2}(t) w^{2}(t) d t\right| \leq$ $E O(C \Delta)$. This relation proves that if $C \Delta$ is sufficiently small, then (26) holds. It follows from (18) that

$$
E_{\mathrm{WS}}=\sum_{k=0}^{K-1} \int_{k \Delta}^{(k+1) \Delta} x^{2}(t) w^{2}(t) d t
$$

By combining (26) and (27), we obtain

$$
E_{\mathrm{WS}} \approx E \sum_{k=0}^{K-1} w^{2}(k \Delta)
$$

Now, we consider the WP estimator. We use (10) to represent $A$ as

$$
A=\frac{1}{T} \sum_{k=0}^{K-1} \int_{k \Delta}^{(k+1) \Delta} w(k \Delta) d t
$$

Hence

$$
A \approx \frac{\Delta}{T} \sum_{k=0}^{K-1} w(k \Delta)=\frac{1}{K} \sum_{k=0}^{K-1} w(k \Delta)
$$

We also rewrite $E_{\mathrm{WP}}$ defined by (24) as $E_{\mathrm{WP}}=$ $\sum_{k=0}^{K-1} \int_{k \Delta}^{(k+1) \Delta} x^{2}(t) w(t) d t$ and approximate it by

$$
E_{\mathrm{WP}} \approx \sum_{k=0}^{K-1} \int_{k \Delta}^{(k+1) \Delta} x^{2}(t) w(k \Delta) d t=E \sum_{k=0}^{K-1} w(k \Delta) .
$$

Similarly to justification of (26), it can be proven that the error in the above approximation is proportional to $O(C \Delta)$. Now, we recall Cauchy's inequality [25]

$$
\left(\sum_{k=0}^{K-1} a_{k} b_{k}\right)^{2} \leq\left(\sum_{k=0}^{K-1} a_{k}^{2}\right)\left(\sum_{k=0}^{K-1} b_{k}^{2}\right)
$$

which holds for any sequences of real numbers $\left\{a_{k}\right\}_{k=0}^{K-1}$ and $\left\{b_{k}\right\}_{k=0}^{K-1}$. By substituting in (22), $a_{k}=1$ and, $b_{k}=w(k \Delta)$, we get

$$
\left(\sum_{k=0}^{K-1} w(k \Delta)\right)^{2} \leq K\left(\sum_{k=0}^{K-1} w^{2}(k \Delta)\right)
$$

Next, we multiply both sides of (33) by $E / K$. This yields

$$
\left(E \sum_{k=0}^{K-1} w(k \Delta)\right)\left(\frac{1}{K} \sum_{k=0}^{K-1} w(k \Delta)\right) \leq\left(E \sum_{k=0}^{K-1} w^{2}(k \Delta)\right) .
$$

By combining (34) with (28), (30), and (31), we obtain

$$
A E_{\mathrm{WP}} \leq E_{\mathrm{WS}}
$$

The conclusion from this analysis is that when the energy of the analyzed signal is uniformly distributed inside the observation window, then the WP method is more accurate than WS.

At this juncture, it is worth to make a comment in relation to WP method. In [7], Bilinskis postulated that DASP ought to be used with sampling schemes for which the point density function is constant. The point density function at time $t_{0}$ is defined as $\lim _{\Delta \rightarrow 0} P_{\left[t_{0}-\Delta / 2, t_{0}+\Delta / 2\right]} / \Delta$, where $P_{\left[t_{0}-\Delta / 2, t_{0}+\Delta / 2\right]}$ is the probability that there is a sampling instant inside the interval $\left[t_{0}-\Delta / 2, t_{0}+\Delta / 2\right]$. Clearly, in the WS approach, the point density function is constant, but in the WP case, the function changes with time proportionally to $w(t)$. Despite this fact, we have constructed an unbiased estimator of the signal spectrum for the WP case, and even more, we have proven that in many practical cases, this estimator is more accurate than WS.

\section{NUMERICAL EXAMPLE OF SPECTRAL ANALYSIS}

In this section, we present a numerical example of applying the proposed methods to spectrum estimation. Our goal is to determine the spectrum of a narrowband signal with unknown spectrum support function (SSF). To achieve this, we examine the estimates of the signal spectrum in a wide range of frequencies. The test signal is $x_{3}(t)=2.13 \times 10^{9} \operatorname{sinc}\left(2.13 \times 10^{9} t\right)-2.11 \times$ $10^{9} \operatorname{sinc}\left(2.11 \times 10^{9} t\right)$. Its spectrum is placed between 1.055 and $1.065 \mathrm{GHz}$. We use a Blackman window of length $T=1 \mu \mathrm{s}$ to define the smoothed spectrum (3) of the truncated signal. The average sampling rate is $1 \mathrm{GHz}$. The spectral analysis of the signal is carried out between DC and 3.5 GHz. The upper limit has been selected quite arbitrarily since the analysis can be performed up to any frequency. Unlike the uniform sampling case, there is no natural upper limit here. The spectrum of the signal is estimated using the WS and WP methods. For comparison, we perform similar analysis of the signal using uniform sampling at the rate of $1 \mathrm{GHz}$. The results are shown in Figs. 1-4. Fig. 1 presents 


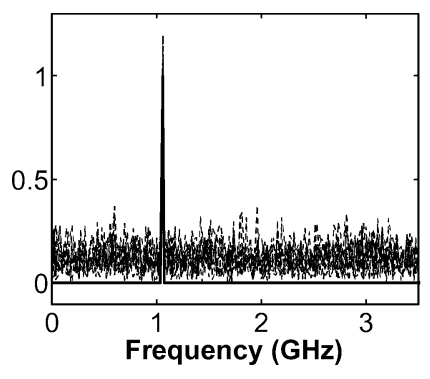

(a)

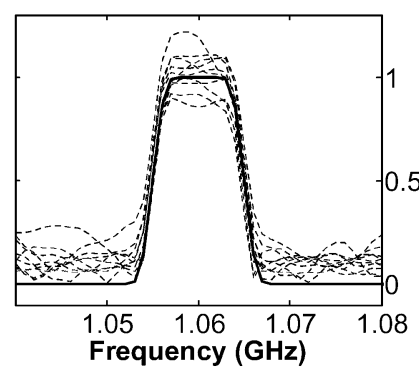

(b)
Fig. 1. Magnitude of $X_{3 W}(f)$ (continuous line) and of its ten WS estimates $X_{3 W S}(f)$ (broken lines). (a) Overall view. (b) Plot zoomed around $1.06 \mathrm{GHz}$.

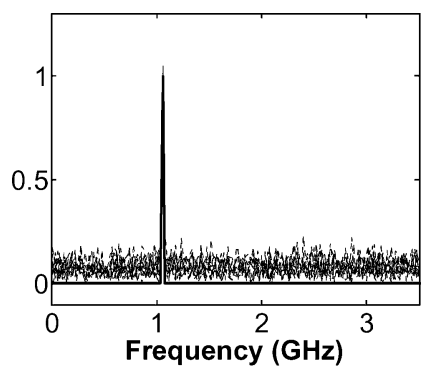

(a)

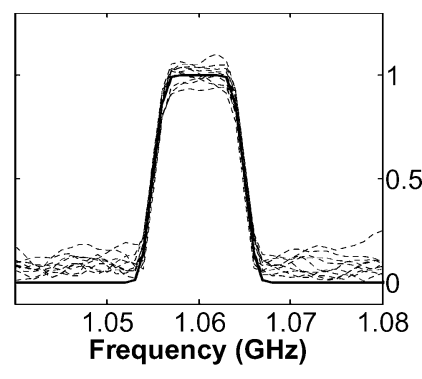

(b)
Fig. 2. Magnitude of $X_{3 W}(f)$ (continuous line) and of its ten WP estimates $X_{3 W P}(f)$ (broken lines). (a) Overall view. (b) Plot zoomed around $1.06 \mathrm{GHz}$.

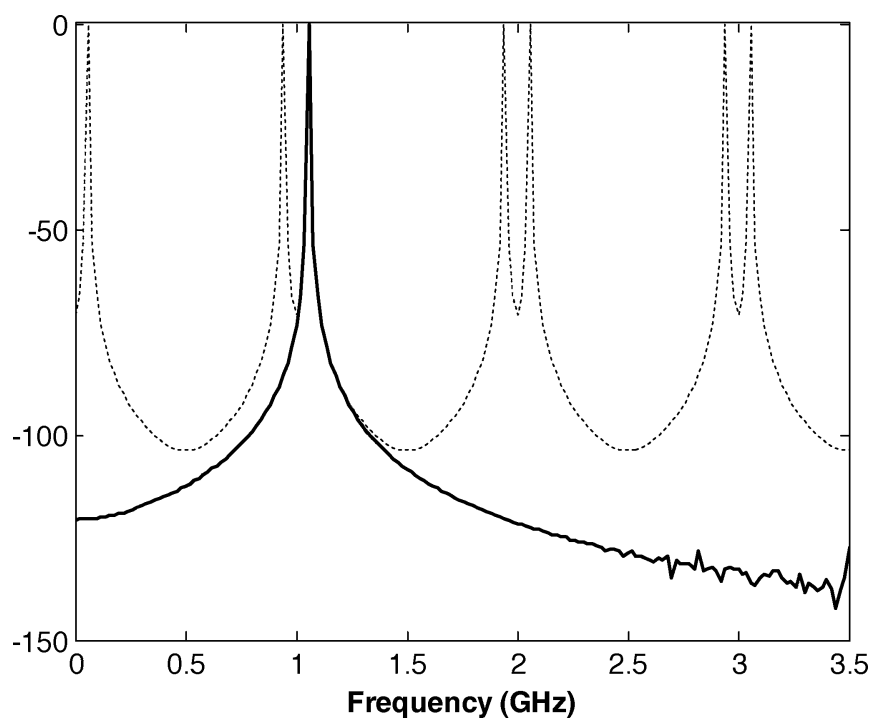

Fig. 3. Magnitude of $X_{3 W}(f)$ (continuous line) and of its DFT-based estimate $X_{3 D F T}(f)$ (broken line).

the magnitude of the signal spectrum $\left|X_{3 W}(f)\right|$ and ten different WS estimates $\left|X_{3 W S}(f)\right|$. Similar results for WP estimation are shown in Fig. 2. Fig. 3 shows the results of spectrum estimation when uniform sampling is used. Owing to aliasing, these results can only be used if the SSF of the analyzed signal is known precisely enough to eliminate spurious components of the estimated spectrum. Fig. 4 shows the standard deviations $\sigma_{\mathrm{WS}}(f)$ and $\sigma_{\mathrm{WP}}(f)$. We also show the plots of RMS errors of the estimated spectra. Each of these errors was obtained by processing results from 1000 independent simulations. Fig. 4 confirms that there is a good match between the theoretical predictions and the outcomes of simulations. It is clear from Fig. 1, 2, and 4 that the

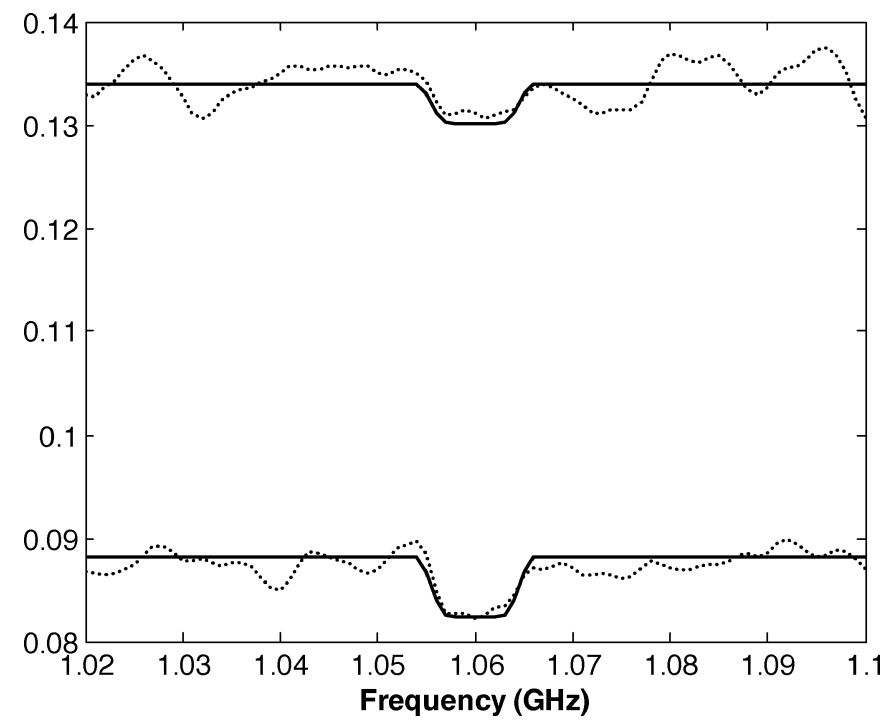

Fig. 4. Magnitude of spectrum estimation errors; RMS WS error (top broken line), $\sigma_{\mathrm{WS}}(f)$ (top continuous line), RMS WP error (bottom broken line), and $\sigma_{\mathrm{WP}}(f)$ (bottom continuous line).

WS and WP methods provide reasonable spectrum estimation at frequencies with strong signal components. At the remaining frequencies, the estimated spectra look like white noise signals whose power densities are determined by the standard deviations of the estimators. According to (21) and (23), these densities can be diminished by increasing the number of samples used in spectral analysis. Plots in Fig. 4 along with (21) and (23) indicate that the WS and WP methods can provide good estimates of the complex spectrum. Therefore, they can be used to approximate not only the magnitude of the spectrum but its phase as well. The above example backs up our claim that the WP approach produces more accurate results than WS. It also confirms that properly used nonuniform sampling eliminates aliasing from digital signal processing.

\section{EFFECT OF SAMPLING INSTANT JitTER ON ACCURACY OF ESTIMATORS}

The analyses performed in the previous sections of this paper were based on the assumption that signal $x(t)$ was sampled exactly at the time instants selected by the random number generator. This assumption is usually not fully satisfied. Every hardware implementation of a sampler suffers from imperfections that result in small time differences between the time instants $\tau_{n}$ when the signal samples are actually taken and the nominal sampling instants $t_{n}$. We refer to these differences as sampling instant jitter, or shortly jitter, and denote them by $\varepsilon_{n}=\tau_{n}-t_{n}$. The effect of jitter on the results of DSP has been studied before [7], [26]. Although the qualitative description of the effects of jitter in those publications is similar to the conclusions we derive here, the quantitative measures cannot be directly applied to our case. These are clearly dependent on the way data is collected and processed, as well as on the objectives to be attained. We show that jitter has at least two undesired effects on spectrum estimation. First, because of jitter, the approximations (5) and (11) are no longer unbiased estimators of $X_{W}(f)$. Fortunately, in many cases, the bias can be removed almost completely. The 
second effect has more serious implications on the practical applicability of the proposed approach. Jitter deteriorates the accuracy of spectrum estimation. This deterioration worsens with increasing frequency. Therefore, for a given level of jitter, there is a practical limitation on how far one can stretch spectral analysis in the frequency domain.

To analyze the above mentioned effects, we note that in the presence of jitter, the WS, and WP spectrum estimators (5) and (11) are replaced with

$$
\begin{aligned}
\hat{X}_{\mathrm{WS}}(f) & \hat{=} \frac{T}{N} \sum_{n=1}^{N} x\left(\tau_{n}\right) w\left(t_{n}\right) \exp \left(-j 2 \pi f t_{n}\right) \\
& =\frac{T}{N} \sum_{n=1}^{N} x\left(t_{n}+\varepsilon_{n}\right) w\left(t_{n}\right) \exp \left(-j 2 \pi f t_{n}\right)
\end{aligned}
$$

and

$$
\begin{aligned}
\hat{X}_{\mathrm{WP}}(f) & \hat{=} \frac{A T}{N} \sum_{n=1}^{N} x\left(\tau_{n}\right) \exp \left(-j 2 \pi f t_{n}\right) \\
& =\frac{A T}{N} \sum_{n=1}^{N} x\left(t_{n}+\varepsilon_{n}\right) \exp \left(-j 2 \pi f t_{n}\right)
\end{aligned}
$$

respectively. In the remainder of this section, we assume that the values $\varepsilon_{n}$ are random variables independent from each other and from the nominal sampling instants $t_{n}, n=1, \cdots, N$. We also assume that all PDFs of $\varepsilon_{n}$ are identical and given by some $p_{\varepsilon}(\varepsilon)$.

We start our analysis by calculating the expected values of estimators $\hat{X}_{\mathrm{WS}}(f)$ and $\hat{X}_{\mathrm{WP}}(f)$ and demonstrating that both are biased. Next, we discuss how the bias can be minimized by constructing modified estimators $\widetilde{X}_{\mathrm{WS}}(f)$ and $\widetilde{X}_{\mathrm{WP}}(f)$ that are practically bias-free. Then, we turn our attention to the variance and standard deviation of $\widetilde{X}_{\mathrm{WS}}(f)$ and $\widetilde{X}_{\mathrm{WP}}(f)$. We conclude this section with practical guidelines on how the size of the jitter limits the range of frequencies within which one may estimate the spectrum of the signal.

To calculate $E\left\{\hat{X}_{\mathrm{WS}}(f)\right\}$, we note that the sum in (36) is composed of $N$ identically distributed random variables. The expected value of each such component, taken with respect to the sampling instant $t$ and time jitter $\varepsilon$, is given by

$$
\begin{aligned}
\hat{X}_{\mathrm{WS}}^{C}(f) \hat{=} & \mathrm{E}\{x(t+\varepsilon) w(t) \exp (-j 2 \pi f t)\} \\
= & \int_{-\infty}^{\infty} \int_{0}^{T} x(t+\varepsilon) w(t) \\
& \quad \times \exp (-j 2 \pi f t) \frac{1}{T} p_{\varepsilon}(\varepsilon) d t d \varepsilon .
\end{aligned}
$$

By substituting $t=\tau-\varepsilon$, we get

$$
\begin{aligned}
\hat{X}_{\mathrm{WS}}^{C}(f)=\frac{1}{T} & \int_{-\infty}^{\infty} p_{\varepsilon}(\varepsilon) \exp (j 2 \pi f \varepsilon) \\
& \times \int_{\varepsilon}^{T+\varepsilon} x(\tau) w(\tau-\varepsilon) \exp (-j 2 \pi f \tau) d \tau d \varepsilon .
\end{aligned}
$$

Further analysis of (39) is performed under the following assumptions.

d) Jitter $\varepsilon$ is significantly smaller than the length of the observation window $T$.

e) The window $w(t) \approx 0$ in the close neighborhood of $t=0$ and $t=T$.

As a consequence of assumptions a)-c) from Section II and d) above, we accept that $w(t)$ satisfies $w(\tau-\varepsilon) \approx w(\tau)$. If we apply this relation to (39), the inner integral can be represented in the following form: $\int_{\varepsilon}^{T+\varepsilon} r(\tau, f) d \tau=\int_{\varepsilon}^{0} r(\tau, f) d \tau+$ $\int_{0}^{T} r(\tau, f) d \tau+\int_{T}^{T+\varepsilon} r(\tau, f) d \tau$, where $r(\tau, f)=x(\tau) w(\tau)$ $\exp (-j 2 \pi f \tau)$. It follows from assumptions $\mathrm{d})$ and e) above that the following relations hold: $\left|\int_{\mathcal{F}}^{0} r(\tau, f) d \tau\right| \ll\left|\int_{0}^{T} r(\tau, f) d \tau\right|$ and $\left|\int_{T}^{T+\varepsilon} r(\tau, f) d \tau\right| \ll\left|\int_{0}^{T} r(\tau, f) d \tau\right|$. These inequalities imply that $\int_{\varepsilon}^{T+\varepsilon} r(\tau, f) d \tau \approx \int_{0}^{T} r(\tau, f) d \tau=\int_{0}^{T} x(\tau) w(\tau)$ $\exp (-j 2 \pi f \tau) d \tau=X_{W}(f)$. Hence, (39) simplifies to

$$
\hat{X}_{\mathrm{WS}}^{C}(f) \approx \frac{\chi(2 \pi f)}{T} X_{W}(f)
$$

where $\chi(\omega) \hat{=} \int_{-\infty}^{\infty} p_{\varepsilon}(\varepsilon) \exp (j \omega \varepsilon) d \varepsilon$ is the characteristic function [25] of the random variable $\varepsilon$. Now, we use (36), (38), and (40) to calculate the expected value of $\hat{X}_{\mathrm{WS}}(f)$

$$
\mathrm{E}\left\{\hat{X}_{\mathrm{WS}}(f)\right\} \approx X_{W}(f) \chi(2 \pi f)
$$

It is visible from (41) that $\hat{X}_{\mathrm{WS}}(f)$ is a biased estimator of signal spectrum. The bias can be approximately removed by replacing $\hat{X}_{\mathrm{WS}}(f)$ with $\widetilde{X}_{\mathrm{WS}}(f)$, which is defined by

$$
\begin{aligned}
\widetilde{X}_{\mathrm{WS}}(f) & \hat{=} \frac{\hat{X}_{\mathrm{WS}}(f)}{\chi(2 \pi f)} \\
& =\frac{T}{\chi(2 \pi f) N} \sum_{n=1}^{N} x\left(\tau_{n}\right) w\left(t_{n}\right) \exp \left(-j 2 \pi f t_{n}\right) .
\end{aligned}
$$

We can perform similar analysis for the WP estimators. The expected value of a single component in summation (37) is given by

$$
\begin{aligned}
\hat{X}_{\mathrm{WP}}^{C}(f) \stackrel{\hat{=}}{=}\{x(t+\varepsilon) \exp (-j 2 \pi f t)\} \\
=\int_{-\infty}^{\infty} \int_{0}^{\infty} x(t+\varepsilon) \\
\quad \quad \times \exp (-j 2 \pi f t) \frac{w(t)}{A T} p_{\varepsilon}(\varepsilon) d t d \varepsilon .
\end{aligned}
$$

By performing calculations similar to those in the WS case, we obtain

$$
\hat{X}_{\mathrm{WP}}^{C}(f) \approx \frac{\chi(2 \pi f)}{A T} X_{W}(f)
$$

Therefore

$$
\mathrm{E}\left\{\hat{X}_{\mathrm{WP}}(f)\right\} \approx X_{W}(f) \chi(2 \pi f) .
$$


An approximately unbiased WP estimator can be constructed as follows:

$$
\widetilde{X}_{\mathrm{WP}}(f) \hat{=} \frac{A T}{\chi(2 \pi f) N} \sum_{n=1}^{N} x\left(\tau_{n}\right) \exp \left(-j 2 \pi f t_{n}\right) .
$$

The above analysis shows that in the presence of sampling instant jitter, our original spectrum estimators are biased. The bias can be approximately removed if the jitter is small in comparison with the length of the window and if the characteristic function of the jitter $\chi(\omega)$ is known.

Now, we analyze the accuracy of the modified estimators $\widetilde{X}_{\mathrm{WS}}(f)$ and $\widetilde{X}_{\mathrm{WP}}(f)$. To this end, we calculate their variance and standard deviation. We show that the size of jitter is a major factor limiting the bandwidth of spectral analysis when using WS or WP approach.

First, we calculate $\widetilde{\sigma}_{X_{\mathrm{WS}}}^{2}(f)$, which is the variance of $\tilde{X}_{\mathrm{WS}}(f)$. The calculations are based on the assumption that both sides of (41) are equal. We start with

$$
\widetilde{\sigma}_{X_{\mathrm{WS}}}^{2}(f)=\mathrm{E}\left\{\left|\widetilde{X}_{\mathrm{WS}}(f)\right|^{2}\right\}-\left|X_{W}(f)\right|^{2} .
$$

Using (42), we get

$$
\begin{aligned}
\mathrm{E}\left\{\left|\widetilde{X}_{\mathrm{WS}}(f)\right|^{2}\right\} & \\
= & \frac{T^{2}}{|\chi(2 \pi f)|^{2} N^{2}} \\
& \times \sum_{n=1}^{N}\left(\sum_{\substack{k=1 \\
k \neq n}}^{N} \mathrm{E}\left\{x^{2}\left(\tau_{n}\right) w^{2}\left(t_{n}\right)\right\}\right. \\
\quad & \mathrm{E}\left\{x\left(\tau_{n}\right) w\left(t_{n}\right) \exp \left(-j 2 \pi f t_{n}\right\}\right. \\
& \times \mathrm{E}\left\{x\left(\tau_{k}\right) w\left(t_{k}\right) \exp \left(j 2 \pi f t_{k}\right\}\right) .
\end{aligned}
$$

We apply (38) and (40) to (48) and obtain

$$
\begin{aligned}
\mathrm{E}\left\{\left|\widetilde{X}_{\mathrm{WS}}(f)\right|^{2}\right\} & =\frac{T^{2}}{|\chi(2 \pi f)|^{2} N^{2}}\left(\mathrm{E}\left\{x^{2}(t+\varepsilon) w^{2}(t)\right\}\right. \\
& \left.+|\chi(2 \pi f)|^{2} \frac{N(N-1)}{T^{2}}\left|X_{W}(f)\right|^{2}\right) .
\end{aligned}
$$

Note that

$$
\mathrm{E}\left\{x^{2}(t+\varepsilon) w^{2}(t)\right\}=\frac{1}{T} \int_{-\infty}^{\infty} p_{\varepsilon}(\varepsilon) \int_{0}^{T} x^{2}(t+\varepsilon) w^{2}(t) d t d \varepsilon .
$$

It follows from assumptions a)-e) that $\int_{0}^{T} x^{2}(t+\varepsilon) w^{2}(t) d t \approx$ $\int_{0}^{T} x^{2}(\tau) w^{2}(\tau) d \tau=E_{\mathrm{Ws}}$. Hence

$$
\mathrm{E}\left\{x^{2}(t+\varepsilon) w^{2}(t)\right\} \approx \frac{E_{\mathrm{WS}}}{T} \int_{-\infty}^{\infty} p_{\varepsilon}(\varepsilon) d \varepsilon=\frac{E_{\mathrm{WS}}}{T} .
$$

After substituting this relation into (49), we get

$$
\mathrm{E}\left\{\left|\widetilde{X}_{\mathrm{WS}}(f)\right|^{2}\right\}=\frac{N-1}{N}\left|X_{W}(f)\right|^{2}+\frac{T E_{\mathrm{WS}}}{|\chi(2 \pi f)|^{2} N}
$$

By combining (52) with (47), we obtain the variance of $\widetilde{X}_{\mathrm{WS}}(f)$

$$
\tilde{\sigma}_{X_{\mathrm{WS}}}^{2}(f)=\frac{T E_{\mathrm{WS}}}{|\chi(2 \pi f)|^{2} N}-\frac{\left|X_{W}(f)\right|^{2}}{N}
$$

and its standard deviation

$$
\tilde{\sigma}_{\mathrm{WS}}(f)=\sqrt{\frac{\frac{T E_{\mathrm{WS}}}{|\chi(2 \pi f)|^{2}}-\left|X_{W}(f)\right|^{2}}{N}} .
$$

The variance $\widetilde{\sigma}_{X_{\mathrm{WS}}}^{2}(f)$ is related to $\sigma_{X_{\mathrm{WS}}}^{2}(f)$ as follows:

$$
\widetilde{\sigma}_{X_{\mathrm{WS}}}^{2}(f)=\sigma_{X_{\mathrm{WS}}}^{2}(f)+\frac{T E_{\mathrm{WS}}}{N} \frac{1-|\chi(2 \pi f)|^{2}}{|\chi(2 \pi f)|^{2}} .
$$

Derivation of the variance and standard deviation of $\widetilde{X}_{\mathrm{WP}}(f)$ can be done in a similar manner. We start with

$$
\widetilde{\sigma}_{X_{\mathrm{WP}}}^{2}(f)=\mathrm{E}\left\{\left|\widetilde{X}_{\mathrm{WP}}(f)\right|^{2}\right\}-\left|X_{W}(f)\right|^{2}
$$

It follows from (46) that

$$
\begin{aligned}
& \mathrm{E}\left\{\left|\widetilde{X}_{\mathrm{WP}}(f)\right|^{2}\right\} \\
& =\frac{A^{2} T^{2}}{|\chi(2 \pi f)|^{2} N^{2}} \\
& \quad \times \sum_{n=1}^{N}\left(\sum_{\substack{k=1 \\
k \neq n}}^{N} \mathrm{E}\left\{x^{2}\left(\tau_{n}\right)\right\}+\mathrm{E}\left\{x\left(\tau_{n}\right) \exp \left(-j 2 \pi f t_{n}\right\}\right.\right. \\
& \quad \times \mathrm{E}\left\{x\left(\tau_{k}\right) \exp \left(j 2 \pi f t_{k}\right\}\right) .
\end{aligned}
$$

By using (43) and (44), we get

$$
\begin{aligned}
\mathrm{E}\left\{\left|\tilde{X}_{\mathrm{WP}}(f)\right|^{2}\right\} & \\
=\frac{A^{2} T^{2}}{|\chi(2 \pi f)|^{2} N}( & \mathrm{E}\left\{x^{2}(t+\varepsilon)\right\}+|\chi(2 \pi f)|^{2} \\
& \left.\times \frac{(N-1)}{A^{2} T^{2}}\left|X_{W}(f)\right|^{2}\right) .
\end{aligned}
$$

Note that $\mathrm{E}\left\{x^{2}(t+\varepsilon)\right\}=(1 / A T) \int_{-\infty}^{\infty} p_{\varepsilon}(\varepsilon) \int_{0}^{T} x^{2}(t+$ $\varepsilon) w(t) d t d \varepsilon \approx\left(E_{\mathrm{WP}} / A T\right)$. After substituting this relation into (58), we have $\mathrm{E}\left\{\left|\widetilde{X}_{\mathrm{WP}}(f)\right|^{2}\right\}=((N-1) / N)\left|X_{W}(f)\right|^{2}+$ $\left(A T E_{\mathrm{WP}} /|\chi(2 \pi f)|^{2} N\right)$. The combination of this formula and (56) yields the variance of $\widetilde{X}_{\mathrm{WP}}(f)$

$$
\widetilde{\sigma}_{X_{\mathrm{WP}}}^{2}(f)=\frac{A T E_{\mathrm{WP}}}{|\chi(2 \pi f)|^{2} N}-\frac{\left|X_{W}(f)\right|^{2}}{N}
$$


TABLE I

CHARACTERISTIC FUNCTIONS OF SOME RANDOM VARIABLES WITH ZERO-SYMMETRIC PDFS

\begin{tabular}{|c|c|c|c|c|}
\hline Jitter & $\mathrm{PDF}-p_{\varepsilon}(\varepsilon)$ & $\sigma_{\varepsilon}$ & $\chi(2 \pi f)$ & $\bar{\chi}\left(f_{\sigma}\right)$ \\
\hline Binary & 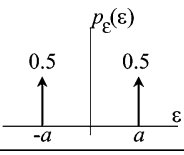 & $a$ & $\cos (2 \pi f a)$ & $\cos \left(2 \pi f_{\sigma}\right)$ \\
\hline Trinary & 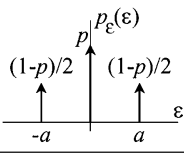 & $a \sqrt{1-p}$ & $p+(1-p) \cos (2 \pi f a)$ & $p+(1-p) \cos \left(\frac{2 \pi f_{\sigma}}{\sqrt{1-p}}\right.$ \\
\hline Uniform & \begin{tabular}{c|c}
$0.5 / a$ & $p_{\varepsilon}(\varepsilon)$ \\
$\square_{-a}$ & \\
\end{tabular} & $\frac{a}{\sqrt{3}}$ & $\frac{\sin (2 \pi f a)}{2 \pi f a}$ & $\frac{\sin \left(2 \sqrt{3} \pi f_{\sigma}\right)}{2 \sqrt{3} \pi f_{\sigma}}$ \\
\hline Triangular & $\int_{-a}^{1 / a} \overbrace{a}^{p_{\varepsilon}(\varepsilon)}$ & $\frac{a}{\sqrt{6}}$ & {$\left[\frac{\sin (\pi f a)}{\pi f a}\right]^{2}$} & {$\left[\frac{\sin \left(\sqrt{6} \pi f_{\sigma}\right)}{\sqrt{6} \pi f_{\sigma}}\right]^{2}$} \\
\hline Normal & $\frac{1}{\sigma \sqrt{2 \pi}} e^{-\frac{\varepsilon^{2}}{2 \sigma^{2}}}$ & $\sigma$ & $e^{-2(\pi f \sigma)^{2}}$ & $e^{-2\left(\pi f_{\sigma}\right)^{2}}$ \\
\hline
\end{tabular}

and its standard deviation

$$
\tilde{\sigma}_{X_{\mathrm{WP}}}(f)=\sqrt{\frac{\frac{A T E_{\mathrm{WP}}}{|\chi(2 \pi f)|^{2}}-\left|X_{W}(f)\right|^{2}}{N}} .
$$

The variance $\widetilde{\sigma}_{X_{\mathrm{WP}}}^{2}(f)$ is related to $\sigma_{X_{\mathrm{WP}}}^{2}(f)$ as follows:

$$
\widetilde{\sigma}_{X_{\mathrm{WP}}}^{2}(f)=\sigma_{X_{\mathrm{WP}}}^{2}(f)+\frac{A T E_{\mathrm{WP}}}{N} \frac{1-|\chi(2 \pi f)|^{2}}{|\chi(2 \pi f)|^{2}} .
$$

One of the elementary properties of the characteristic function $\chi(\omega)$ is that its magnitude never exceeds one [25]. Therefore, it follows from (55) and (61) that $\widetilde{X}_{\mathrm{WS}}(f)$ and $\widetilde{X}_{\mathrm{WP}}(f)$ have larger variance and standard deviation than those of $X_{\mathrm{WS}}(f)$ and $X_{\mathrm{WP}}(f)$.

Similarly to the jitter-free case, we can determine for each method the maximum value of the variance. In the WS case, we have

$$
\widetilde{\sigma}_{\mathrm{WS}}(f) \leq \widetilde{\sigma}_{\mathrm{WS}, \max }(f) \hat{=} \frac{1}{|\chi(2 \pi f)|} \sqrt{\frac{T E_{\mathrm{WS}}}{N}}
$$

while for the WP method

$$
\widetilde{\sigma}_{\mathrm{WP}}(f) \leq \widetilde{\sigma}_{\mathrm{WP}, \max }(f) \hat{=} \frac{1}{|\chi(2 \pi f)|} \sqrt{\frac{A T E_{\mathrm{WP}}}{N}} .
$$

In order to better understand how jitter deteriorates the quality of spectrum estimation, we present Table I, which contains characteristic functions of some random variables with zero-symmetric PDFs. These functions are then modified to relate them to "standardized frequency" $f_{\sigma}=f \sigma_{\varepsilon}$, where $\sigma_{\varepsilon}$ is the standard deviation of the jitter. The modified version is defined by $\bar{\chi}\left(f_{\sigma}\right) \hat{=} \chi\left(2 \pi f_{\sigma} / \sigma_{\varepsilon}\right)$. Fig. 5 shows the plots of the magnitudes of $\bar{\chi}\left(f_{\sigma}\right)$. It is clear from the plot that for small values of the standardized frequency (say $f_{\sigma}<0.1$ ), the functions $\bar{\chi}\left(f_{\sigma}\right)$ do not differ very much from each other. In fact, they

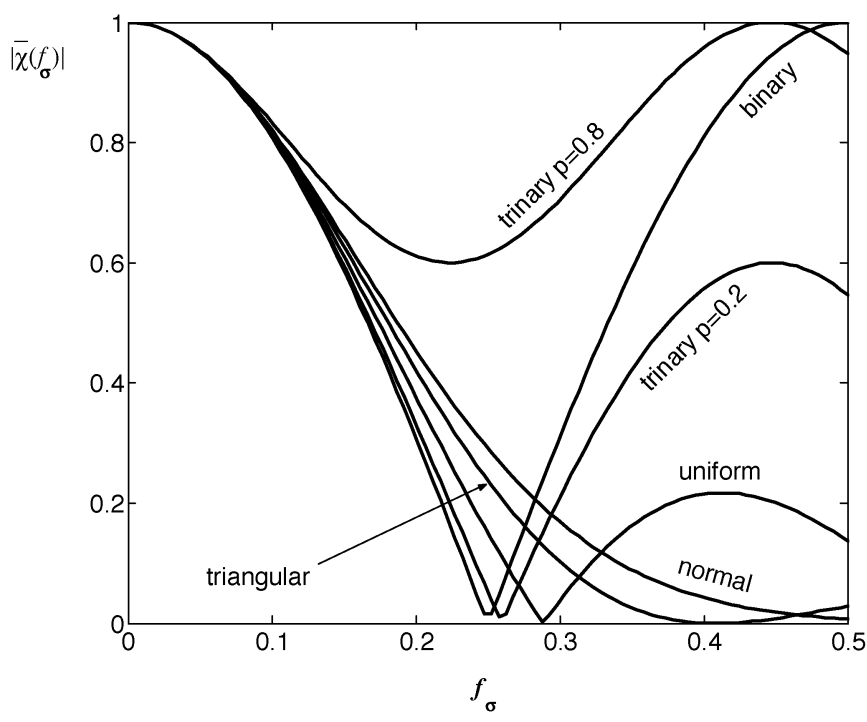

Fig. 5. Magnitude of $\bar{\chi}\left(f_{\sigma}\right)$ for selected types of sampling instant jitter.

can be described by their partial Taylor series expansion [25] $\bar{\chi}\left(f_{\sigma}\right) \approx 1-2 \pi^{2} f_{\sigma}^{2}$. As long as this approximation holds, the bias introduced by jitter can be compensated whenever $\sigma_{\varepsilon}$ is known. Detailed knowledge of the PDF is no longer needed. It should be noted that the above simplification does not hold in the case of impulsive jitter-occasional bursts of relatively large jitter followed by quiet periods of small jitter. In such cases, our second-order polynomial approximation of $\bar{\chi}\left(f_{\sigma}\right)$ remains valid in an extremely narrow range of frequencies around DC, and the biased estimators cannot be compensated without knowledge of $\chi(\omega)$ or higher order moments of the jitter.

Formulas (54) and (60) provide a basis for determining the bandwidth analysis of spectrum estimation that uses the WS and WP approaches. It is clear from Fig. 5 that deterioration of standard deviations of WS and WP estimators caused by jitter 


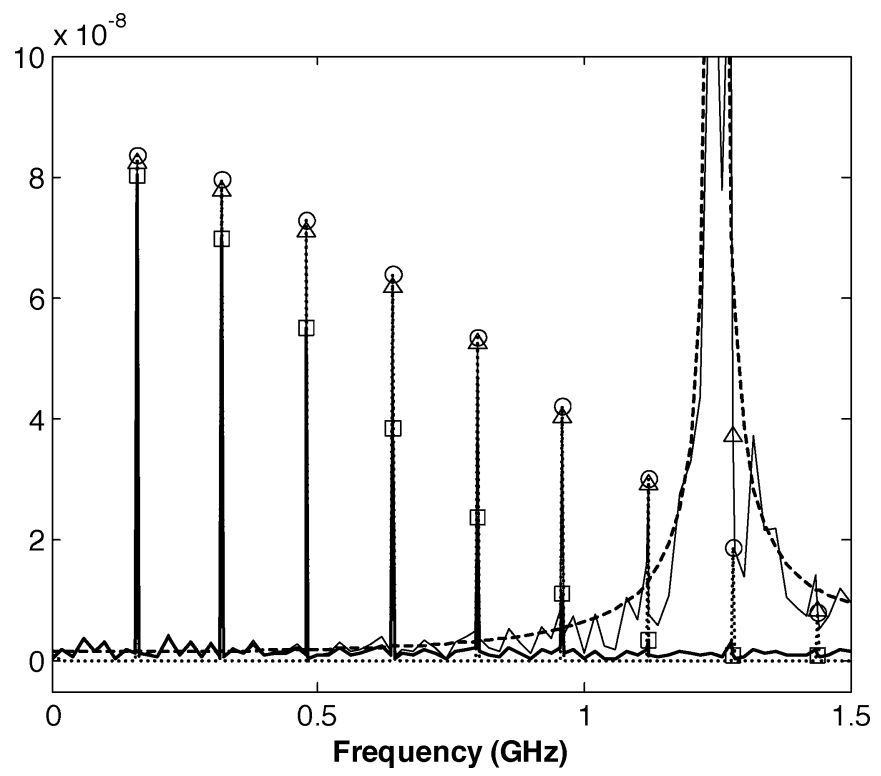

Fig. 6. WS spectrum estimation with jitter: Spectrum of the continuous-time signal $\left|X_{4 W}(f)\right|$ (dotted line, circles), the average of 200 uncompensated WP estimates $\left|\hat{X}_{4 W S}(f)\right|$ (thick continuous line, squares), the average of 200 compensated WS estimates $\left|\widetilde{X}_{4 W S}(f)\right|$ (thin continuous line, triangles), and $\widetilde{\sigma}_{\mathrm{WS}, \max }(f) / \sqrt{200}$ (broken line).

is not very significant, as long as the standardized frequency is small. The influence of the $\left|X_{W}(f)\right|^{2}$ component on the values of the standard deviations $\widetilde{\sigma}_{X_{\mathrm{WS}}}(f)$ and $\widetilde{\sigma}_{X_{\mathrm{WP}}}(f)$ is often negligible. Therefore, $\widetilde{\sigma}_{X_{\mathrm{WS}}}(f)$ and $\widetilde{\sigma}_{X_{\mathrm{WP}}}(f)$ can be considered to be inversely proportional to $|\chi(2 \pi f)|$. If we conservatively demand that, e.g., $|\chi(2 \pi f)|>0.8$, then it follows from Fig. 5 that $f_{\sigma}<0.1$, and the bandwidth of spectral analysis becomes directly related to the standard deviation of jitter: $f_{\max }=0.1 / \sigma_{\varepsilon}$. Note that this limit is only indicative and does not constitute a definite boundary for spectral analysis.

\section{NUMERICAL ANALYSIS OF THE EFFECTS OF JitTER}

Simulation results presented in this section illustrate how the sampling instant jitter affects the bandwidth within which spectral analysis can be effectively performed. The test signal used here is a zero-mean nonsymmetric square wave of frequency $f_{0}=160 \mathrm{MHz}$ defined by

$$
x_{4}(t)= \begin{cases}1.8, & \text { when } \bmod (t, P) \in[0,0.1 P] \\ -0.2, & \text { when } \bmod (t, P) \in(0.1 P, P]\end{cases}
$$

where $P=1 / f_{0}$ is the period of $x_{4}(t)$. We define the spectrum (3) of $x_{4}(t)$ with the use of a Blackman window. The results of spectrum estimation with the WS method are shown in Fig. 6 and with the WP method in Fig. 7. In each case, we show the magnitude of the target spectrum and of the estimated spectra with and without jitter compensation. These results of spectrum estimation are averaged over 200 independent experiments. We also plot $\widetilde{\sigma}_{\mathrm{WS}, \max }(f) / \sqrt{200}$ and $\widetilde{\sigma}_{\mathrm{WP}, \max }(f) / \sqrt{200}$. The scaling factor $\sqrt{200}$ was introduced to show the standard deviation of the averaged estimations. The sampling frequency used for WS and WP estimation was $250 \mathrm{MHz}$, and the length of the observation window was $1 \mu \mathrm{s}$. The PDF of the sampling instant jitter in these experiments was distributed uniformly in

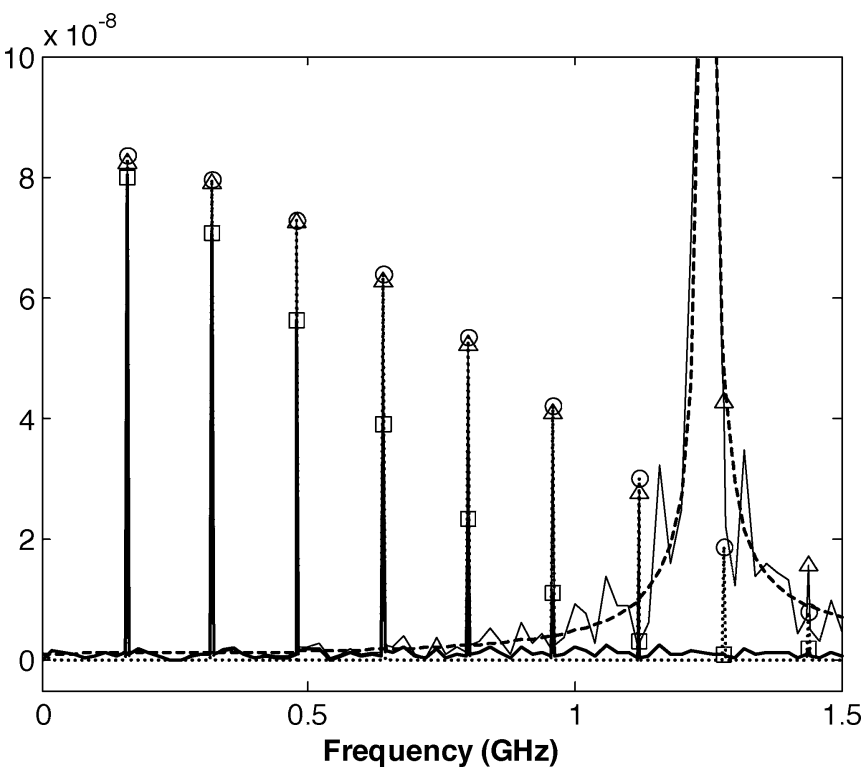

Fig. 7. WP spectrum estimation with jitter: Spectrum of the continuous-time signal $\left|X_{4 W}(f)\right|$ (dotted line, circles), the average of 200 uncompensated WP estimates $\left|\hat{X}_{4 W P}(f)\right|$ (thick continuous line, squares), the average of 200 compensated WP estimates $\left|\widetilde{X}_{4 W P}(f)\right|$ (thin continuous line, triangles), and $\widetilde{\sigma}_{\mathrm{WP}, \max }(f) / \sqrt{200}$ (broken line).

the interval $\left[-4 \times 10^{-10}, 4 \times 10^{-10}\right] \mathrm{s}$. Note that without jitter compensation, the bias of spectrum estimation can be ignored only at low frequencies. At higher frequencies, the estimated spectrum tends to be weaker than the true one, whereas the noise-resembling component of the estimated spectrum remains unchanged. This can be interpreted as deterioration of the signal-to-noise ratio with increasing frequency. When jitter is compensated, the bias disappears, but the poor signal-to-noise ratio at high frequencies is not affected by this compensation. This observation confirms that jitter imposes a practical limit on the bandwidth of spectral analysis.

\section{CONCLUSIONS}

The proposed WS and WP algorithms allow digital alias-free estimation of signal spectrum. These algorithms use specific probability density functions to randomly select sampling instants. The estimates of the spectrum are calculated using summation formulas similar to the nonuniform DFT. These formulas are appropriately adjusted to match the sampling scheme used by each method. The theoretical and experimental studies have shown that both methods provide unbiased estimates of signal spectrum. Two factors imposing limits on the use of the proposed methods have been investigated in this paper: the level of the spectrum estimation error and the sampling instant jitter.

For each method, the spectrum estimation error resembles a wideband noise. Its power density is practically constant except at frequencies at which the analyzed signal is strong. At these places, the power density of the noise is slightly lower. The adverse effect of the noise is that the spectral components of the analyzed signal whose spectrum does not exceed its level are masked and can hardly be detected by the proposed methods.

The second limiting factor (the jitter) introduces bias to spectrum estimation. In many cases, this bias can be almost 
completely removed. The side effect of this operation is increased level of spectrum estimation errors at higher frequencies. This phenomenon effectively limits the bandwidth within which spectral analysis can be performed. A simple analysis shown here suggests that the bandwidth of spectral estimation is limited by approximately $10 \%$ of the inverse of the standard deviation of the jitter. It should be noted that in practical implementation of the proposed methods, other limiting factors might emerge. For example, if all the sampling instants created by the pseudorandom generator are multiples of some small number $\Delta T$, then the limit of $0.5 / \Delta T \mathrm{~Hz}$ is also imposed on the bandwidth.

\section{ACKNOWLEDGMENT}

The authors thank D. Thompson for his valuable comments that helped improve the final version of the paper.

\section{REFERENCES}

[1] F. Marvasti, A Unified Approach to Zero-Crossing and Nonuniform Sampling of Single and Multidimensional Signals and Systems. Oak Park, IL: Nonuniform, 1987.

[2] — Nonuniform Sampling, Theory and Practice. New York: Kluwer/Plenum, 2001.

[3] J. L. Yen, "On nonuniform sampling of bandwidth-limited signals," IRE Trans. Circuit Theory, vol. CT-3, pp. 251-257, Dec. 1956.

[4] D. J. Wingham, "The reconstruction of a band-limited function and its Fourier transform from a finite number of samples at arbitrary locations by singular value decomposition," IEEE Trans. Signal Processing, vol. 40, pp. 559-570, Mar. 1992.

[5] P. J. S. G. Ferreira, "Incomplete sampling and the recovery of missing samples from oversampled band-limited signals," IEEE Trans. Signal Processing, vol. 40, pp. 225-227, Jan. 1992.

[6] G. B. Irvine and D. R. S. Cumming, "Algorithms for the nonuniform acquisition and subsequent reconstruction of data," in Proc. 14th Int. Conf. Digital Signal Process., 2002, pp. 791-794.

[7] I. Bilinskis and M. Mikelsons, Randomized Signal Processing. London, U.K.: Prentice-Hall, 1992.

[8] H. S. Shapiro and R. A. Silverman, "Alias-free sampling of random noise," SIAM J. Appl. Math., vol. 8, pp. 225-236, June 1960.

[9] E. Masry, "Alias-free sampling: an alternative conceptualization and its applications," IEEE Trans. Inform. Theory, vol. IT-24, pp. 317-324, May 1978.

[10] K.-S. Lii, "Alias-free cross bispectral analysis of continuous-time vector processes under sampling," in Proc. Int. Workshop Sampling Applicat., 1997, pp. 233-238.

[11] I. Bilinskis and A. Mikelsons, "Application of randomized or irregular sampling as an anti-aliasing technique," in Signal Processing V: Theories and Applications, L. Torres, E. Masgrau, and M. A. Lagunas, Eds. New York: Elsevier, 1990, pp. 505-508.

[12] Y. Artyukh, I. Bilinskis, M. Greitans, and V. Vedin, "Signal digitizing and recording in the DASP-Lab system," in Proc. Int. Workshop Sampling Applicat., 1997, pp. 357-360.

[13] J. J. Wojtiuk, "Randomised sampling for radio design," Ph.D. dissertation, Univ. South Australia, Mawson Lakes, Australia, 2000.

[14] R. J. Martin, "Irregularly sampled signals: Theories and techniques for analysis," Ph.D. dissertation, Univ. College London, London, I.K., 1998.

[15] A. Tarczynski, "Spectrum estimation of nonuniformly sampled signals," in Proc. 14th Int. Conf. Digital Signal Process., 2002, pp. 795-798.
[16] D. M. Bland, T. Laakso, and A. Tarczynski, "Analysis of algorithms for nonuniform-time discrete Fourier transform," in Proc. Int. Symp. Circuits Syst., vol. 2, 1996, pp. 453-456.

[17] N. R. Lomb, "Least-squares frequency analysis of unequally spaced data," Astrophys. Space Sci., vol. 39, pp. 447-462, Feb. 1976.

[18] J. D. Scargle, "Studies in astronomical time series analysis. II. Statistical aspects of spectral analysis of unevenly spaced data," Astronomical J., vol. 263, pp. 835-853, Dec. 1982.

[19] _ - "Studies in astronomical time series analysis. III. Fourier transforms, autocorrelation functions, and cross-correlation functions of unevenly spaced data," Astronomical J., vol. 343, pp. 874-887, Aug. 1989.

[20] I. Bilinskis and G. D. Cain, "Digital alias-free signal processing in the $\mathrm{GHz}$ frequency range," in IIE Colloq. Advanced Signal Process. Microwave Applicat., Nov. 1996, Dig. 1996/226, pp. 6/1-6/6.

[21] I. Mednieks, "Methods for spectral analysis of nonuniformly sampled signals," in Proc. Workshop Sampling Theory Applicat., 1999, pp. 190-193.

[22] Y. Artyukh, A. Ribakov, and V. Vedin, "Evaluation of pseudorandom sampling processes," in Proc. Workshop Sampling Theory Applicat., 1997, pp. 361-363.

[23] A. Antoniou, Digital Filters: Analysis, Design and Applications. New York: McGraw-Hill, 1993.

[24] A. G. Deczky, "Unispherical windows," in Proc. IEEE Int. Symp. Circuits Syst., 2001, pp. 85-88.

[25] E. W. Weisstein, CRC Encyclopedia of Mathematics. London, U.K.: CRC, 1999.

[26] A. Berkovitz and I. Rusnak, "FFT processing of randomly sampled harmonic signals," IEEE Trans. Signal Processing, vol. 40, pp. 2816-2819, Nov. 1992.

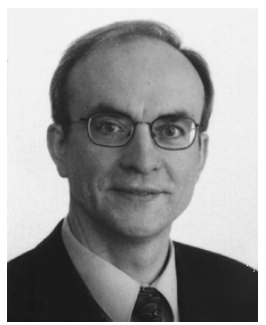

Andrzej Tarczynski (M’01) received the M.Eng. and $\mathrm{Ph} . \mathrm{D}$. degrees in automatic control from Warsaw University of Technology (WUT), Warsaw, Poland, in 1979 and 1986, respectively.

From 1981 to 1990 , he was a lecturer and researcher with the Institute of Control and Industrial Electronics, WUT. In 1991, he joined the University of Westminster (UoW), London, U.K. Currently he is a Reader in signal processing and control systems at the Department of Electronic Systems, UoW. His research interests concentrate around various aspects of the theory of systems, especially in the area of signal processing and control engineering.

Dr. Tarczynski is a member of IEE.

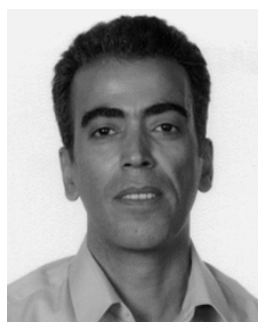

Najib Allay received the B.Eng. (Hons.) degree in control and computer engineering in 2000 from the University of Westminster, London, U.K., and the M.Sc. degree in communication systems and signals processing from the University of Bristol, Bristol, U.K., in 2001. He is currently pursuing the Ph.D. degree with the Department of Electronic Systems, University of Westminster.

His research interests include nonuniform sampling and signal processing for communications with focus on software-defined radio.

Mr. Allay is a member of IEE. 